\title{
Rapid characterisation of potentially hazardous blocks in open pit mining
}

\author{
M.K. Elmouttie CSIRO Earth Science and Resource Engineering, Australia \\ G.V. Poropat CSIRO Earth Science and Resource Engineering, Australia \\ G. Krähenbühl CSIRO Earth Science and Resource Engineering, Australia \\ P. Dean CSIRO Earth Science and Resource Engineering, Australia
}

\begin{abstract}
The management of open pit mine slope stability analysis can be optimised by using all available data ranging from qualitative assessment of rock mass properties through to the results of sophisticated computer-based analyses. The choice of approach is typically based on both technical and resource constraints. In this paper we propose a practical method to manage design risks by understanding the potential structure-controlled failure mechanisms of a slope. In particular we emphasise that an understanding of the trustworthiness or level of confidence associated with the stability analysis is essential to manage the rock mass and to determine the appropriate amount of resources required.
\end{abstract}

The example discussed in this paper involves consideration of the potential formation of kinematically hazardous blocks on a mine highwall or bench face, where it is not clear from the outset what approach is required. The proposed method provides a quick assessment based on the spatial location of structure data and the practical uncertainties resulting from variation of key input data. In this paper, we will present new methods to provide this guidance based on recent advances in the use of digitally mapped structural data, first pass rigid block stability analysis and analysis of the confidence of the stability analysis undertaken.

\section{Introduction}

As open cut mining operations become deeper, so increases the need to quantitatively evaluate the risk associated with potential geotechnical hazards. For instance, failures in deep pits can occur at a number of scales (i.e. single bench, inter-ramp scale or entire slope). Sophisticated slope monitoring technologies provide mine operators with the ability to continue mining as the behaviour of a slope deteriorates, prior to an eventual failure. However this does not diminish the need to characterise in advance the nature of the failure mechanism. In fact, a better understanding of the mechanism can greatly improve its management as well as design and influence the operational decisions to be made during the evolution of the mine.

Potential slope failure mechanisms can be divided into several broad categories: weak-rock shear failure (heavily jointed or weak intact rock), structurally controlled translational failure, complex translational failure including step-path failure and complex translational/rotational failure including yielding (Stead et al., 2006). The properties of the rock material (stress concentration, fracture properties, etc.) are not static but time dependent and can respond to factors such as heavy rainfall, mining-related stress change and blasting operations. Clearly, the prediction of hazard location, likelihood and significance is a complex problem requiring an understanding of the underlying physics, scale, and uncertainty associated with the rock mass, rock material, and potential failure mechanisms.

Figure 1 illustrates the 'parameter space' for modelling complex problems. The practitioner is required to use 'expert judgment', appropriate design methods and sensitivity analyses to determine how to cover this parameter space in a way that is optimal in terms of model accuracy (i.e. how well it replicates reality) and trustworthiness (how certain the predictions obtained from the model are believed to be) versus resources (time and money). However, too often the uncertainty parameter (trustworthiness) is neglected because 
the tools used by the practitioner do not easily allow its interrogation or because characterisation of the uncertainty is deemed to be too resource intensive.

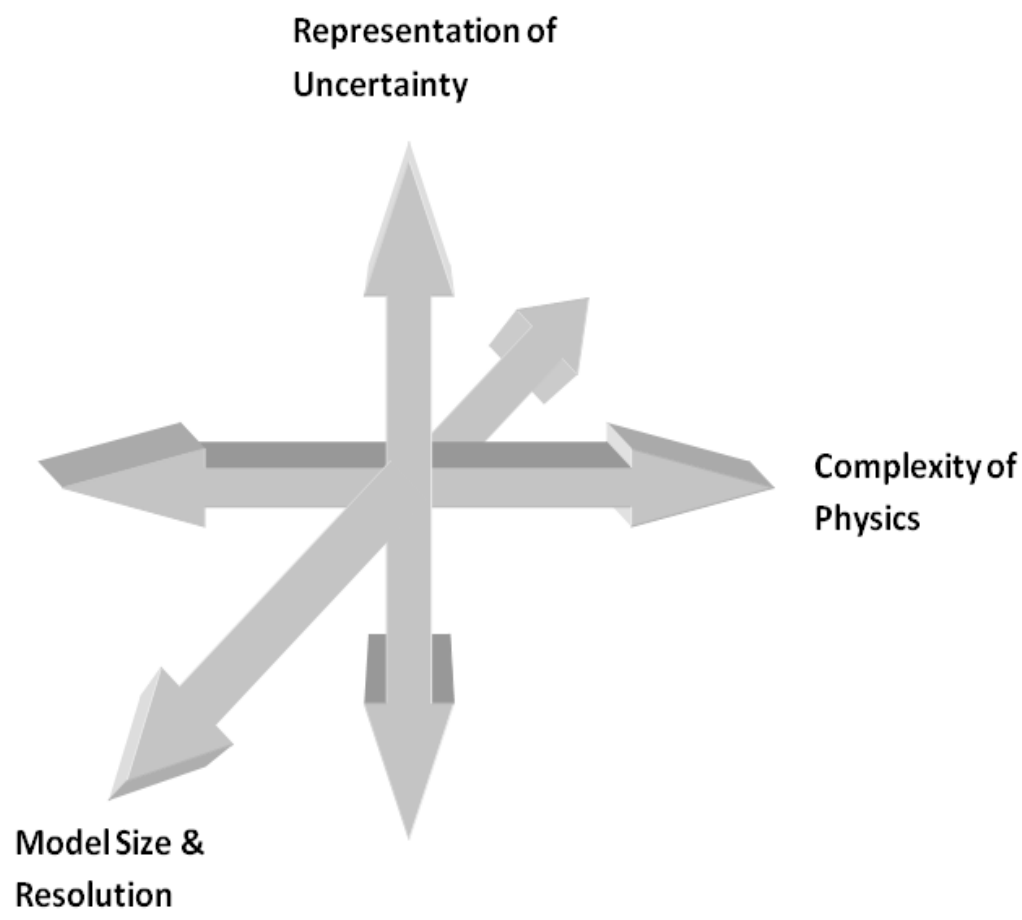

Figure 1 Parameter space for modelling large, complex problems with uncertainty

In this paper, the uncertainty associated with characterisation of structurally controlled hazards is discussed. A method is described to provide guidance to the practitioner regarding the variance in the scope of the parameter space (scale versus physics versus uncertainty) requiring investigation. Recent advances in the use of digitally mapped structural data and statistically based rigid block stability analysis are drawn upon and examples of application of the proposed method are described. Although the scope of the paper is constrained to structurally controlled failures, application of the method to other failure mechanisms is described.

\section{Hazardous blocks}

In this paper, the term 'hazardous block' is used to describe a kinematically free block of rock of significant mass that has the potential to collapse or move in an unplanned manner. Characterisation of blocks, let alone 'potentially hazardous blocks', is not a trivial task. Several questions must be addressed, such as: are blocks in the rock mass defined almost entirely by structures (i.e. blocky rock mass) or is step-path formation of blocks more likely; if the latter, can geometrical methods be used to estimate the properties of the block formation.

The term 'rapid characterisation' is used in this paper to describe a method which allows quantification of the block properties and their stability characteristics within a time frame that is potentially suitable for regular use in mine operations.

\section{Techniques}

Depending on operational considerations the practitioner may be able to use one or more techniques that support rapid characterisation of hazardous blocks. Their relative merits are discussed below.

\subsection{Stereonet analysis}

The most basic, but widely used characterisation technique is kinematic analysis using a stereonet. Traditional analysis using a stereonet is typically applied to the analysis of planar, wedge or toppling failure 
modes. The stereonet can provide the practitioner with an indication of the existence and stability of wedge formation given a bench orientation and the orientation of intersecting structures. However, the analysis assumes persistent structures and takes no account for the relative location of structures and is therefore very conservative in that it essentially may identify blocks that can never exist.

\subsection{Simple wedge analysis}

If a maximum of two dominant structure sets are being considered, and the most geometrically complex block formation under consideration is believed to be a tetrahedral wedge, then a 'simple wedge analysis' can be used. Statistical parameters and probability distribution functions (pdf) can be estimated (but often are assumed) to represent the orientations and surface properties of the sliding planes forming the wedge. A statistical sampling method (e.g. Monte Carlo simulation) can be used to generate multiple wedge realisations. Added data such as excavation geometry and the presence or absence of tension cracks can be used to further constrain the analysis. Such analysis allows the estimation of statistical parameters for Factors of Safety (or probability of failure), volumes (or masses) and other failure parameters.

\subsection{Wedge analysis constrained by digitally mapped structures}

Digital mapping of the excavation allows the spatial location and the observed persistence of structures to be used as further constraints for the wedge analysis. A combinatorial analysis can be used in combination with user inputs defining persistence and surface properties to predict potential wedge formation and stability (Figure 2). Although the statistical significance of such an analysis will be determined by the numbers of structures mapped, this can highlight the inherent uncertainty present in characterising rock mass discontinuities. This uncertainty is also present in the analysis technique discussed in Section 3.2 but may not be apparent to the practitioner because of the use of predefined pdfs.

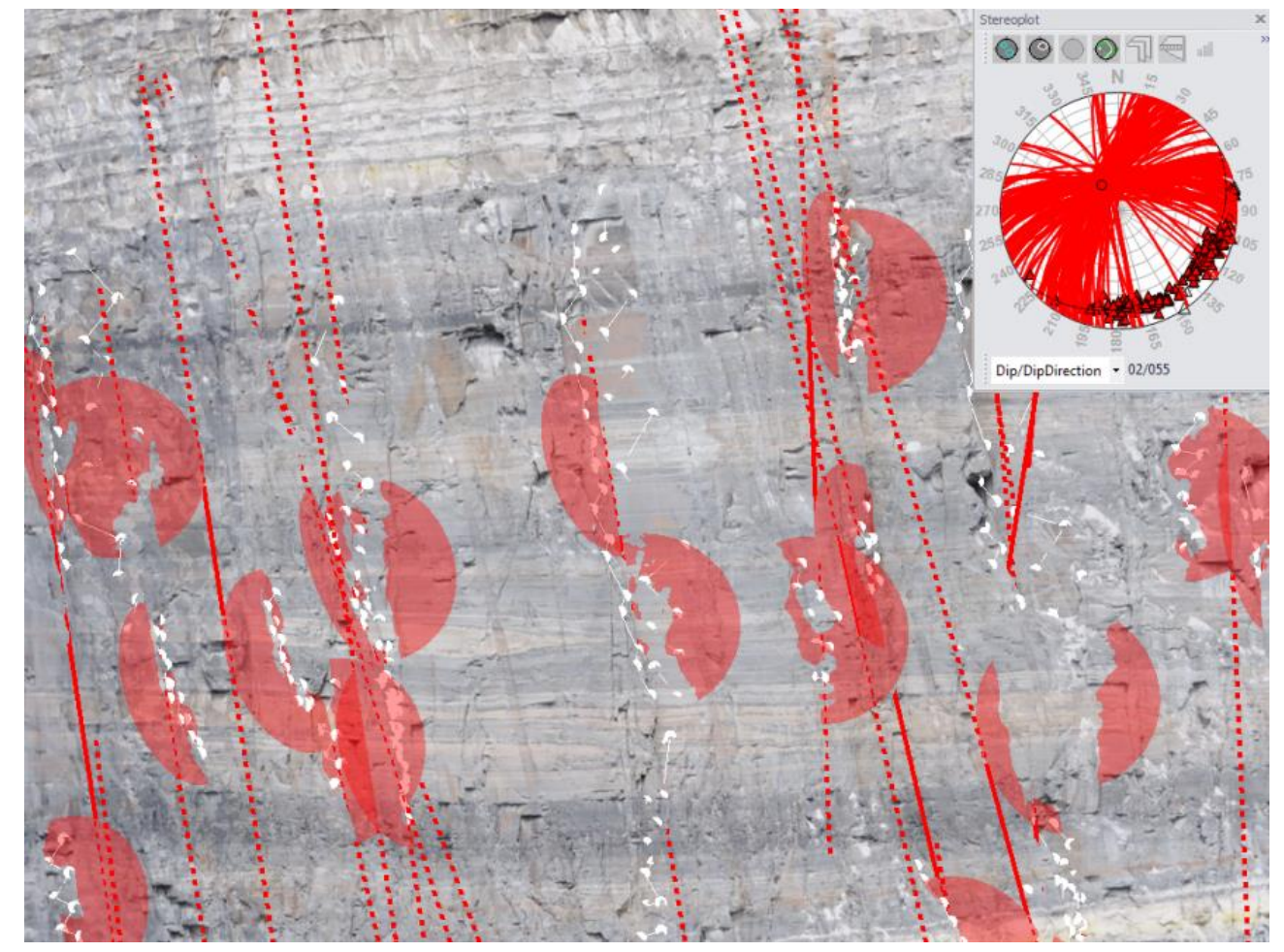

Figure 2 Screenshot showing automated wedge analysis for a popular photogrammetric based geotechnical software package. Sliding planes are shown as red discs and sliding vectors as dotted lines 


\subsection{Polyhedral stability analysis constrained by digitally mapped structures and discrete fracture networks}

For more accurate representation of the in situ blocks in the rock mass, more sophisticated methods are required. Polyhedral stability analysis using discrete fracture networks (DFN) constrained by digitally mapped structures can accommodate any number of structure sets and the polyhedra analysed can have arbitrary numbers of facets. Details of the use of these methods are presented in the following sections. These methods are not as commonly employed as those discussed in previous sections and some understanding of these methods may be required to appreciate the improved hazard assessment they can achieve.

\subsubsection{Discrete fracture network}

The use of discrete geometric representations of fracture/joint networks to model the heterogeneity of a rock mass has greatly increased in recent years. The acceptance of this method has been a function of both the availability of software based DFN generators as well as the widespread availability of suitable computing resources. DFN provide a more realistic simulation of rock mass heterogeneity than do the more established equivalent continuum medium (ECM) methods (such as finite element methods - see Jing, 2003 for a review). ECM methods often rely on assumptions of parallel fractures within a given set, fracture ubiquity, and assumed infinite persistence of fractures.

DFN techniques do not require such unrealistic assumptions and therefore better represent individual fracture properties such as position, size and aperture and (potentially) shape. However, while DFN methods improve the model capability the potential of the DFN approach can only be fully realised if the geometry of the discontinuity network being generated is statistically similar to the in situ network of rock mass defects being simulated. The DFN should also honour the geological history of the structure formation by accurately representing the termination and truncation of structures. If similitude is not achieved, the DFN approach can be considered, at best, to be an inefficient and overly complicated alternative to the ECM approach. At worst, the DFN can misrepresent the characteristic of the network, which has significant implications for the results from stability, fragmentation, hydrogeological and other analyses.

A DFN is developed using both the deterministic structures that have been explicitly measured (e.g. larger structures such as faults or fractures that have been mapped on an exposure) and the stochastic or inferred structures. It is particularly important to include large structures deterministically in a DFN because stochastic generation of large fractures can severely alter the geometries (and subsequent hydrological, stability and other analyses).

The DFN generation process for stochastic structures relies on the ability of the practitioner to create probability distribution functions representative of the fracture parameters such as density, orientation and size. These can only be generated after the statistical parameters of the discontinuity matrix observed in the field have been quantified. Assumptions must also be made regarding the fracture shape (Dershowitz, 1984; Billaux et al., 1989).

A commonly adopted DFN model for the simulated rock mass is the Baecher model (Baecher et al., 1978). This model assumes discontinuities are randomly distributed spatially (i.e. Poisson process), the sizes (persistence) of discontinuities can be described by lognormal or negative-exponential distributions and the spacings between adjacent discontinuities from the same set (as measured by projecting discontinuity centroids along the mean set normal vector) can be described by negative-exponential distributions.

The assumptions used to create any given fracture model may only be adequate for generic studies. When the characteristics of a particular site are being investigated, the individual fracture properties must be estimated. These properties are represented as random variables and therefore statistical methods must be used to generate simulated fracture networks (known as realisations). The method adopted in the research described here is known as Monte Carlo sampling or simulation. 


\subsubsection{Polyhedral modelling}

Identification of the polyhedra or rock blocks present in a rock mass is an important tool in the application of block theory and numerical studies of rock mass stability. Polyhedral modelling does not provide the same level of physics sophistication as numerical stress-strain modelling however it is extremely useful for several reasons. It is compatible with fast, first pass analyses; more advanced than the more traditional analyses described in the preceding sections; compatible with statistical sampling schemes such as Monte Carlo; and is able to provide estimates of in situ block size distribution, slope stability, rock mass fragmentation, etc., when coupled with accurate structural representations.

Used in isolation from numerical techniques, polyhedral modelling will provide an estimate of the 3D fragmentation of a rock mass but will not account for stress induced fracture propagation. Numerous polyhedral modellers have been described in the literature and used for estimation of block size distribution (e.g. Wang et al., 2003; Rogers et al., 2007; Menéndez-Díaz et al., 2009). Traditionally, polyhedral modellers have required simplifications such as cubic or hexahedral representations of the simulation volume; very small numbers of discontinuities; the assumption of planar topologies for the discontinuities; and the assumption of infinite persistence.

The third and fourth simplifications impose a critical limit on the ability to represent a rock mass comprising irregular shaped blocks or polyhedra. In particular, infinitely persistent discontinuities disallow the inclusion of concave extremities and can only yield convex polyhedra. These limitations may result in significant errors when assessing the stability of an excavation (Elmouttie et al., 2008) and in situ block size distribution (Elmouttie et al., 2009). The polyhedral modeller utilised in this project represents an evolution of modellers outlined in (Lin et al., 1987; Jing and Stephansson, 1994; Jing, 2000; Lu, 2002) and is described in detail in Elmouttie et al., 2010. Examples of polyhedral models are shown in Figure 3. 


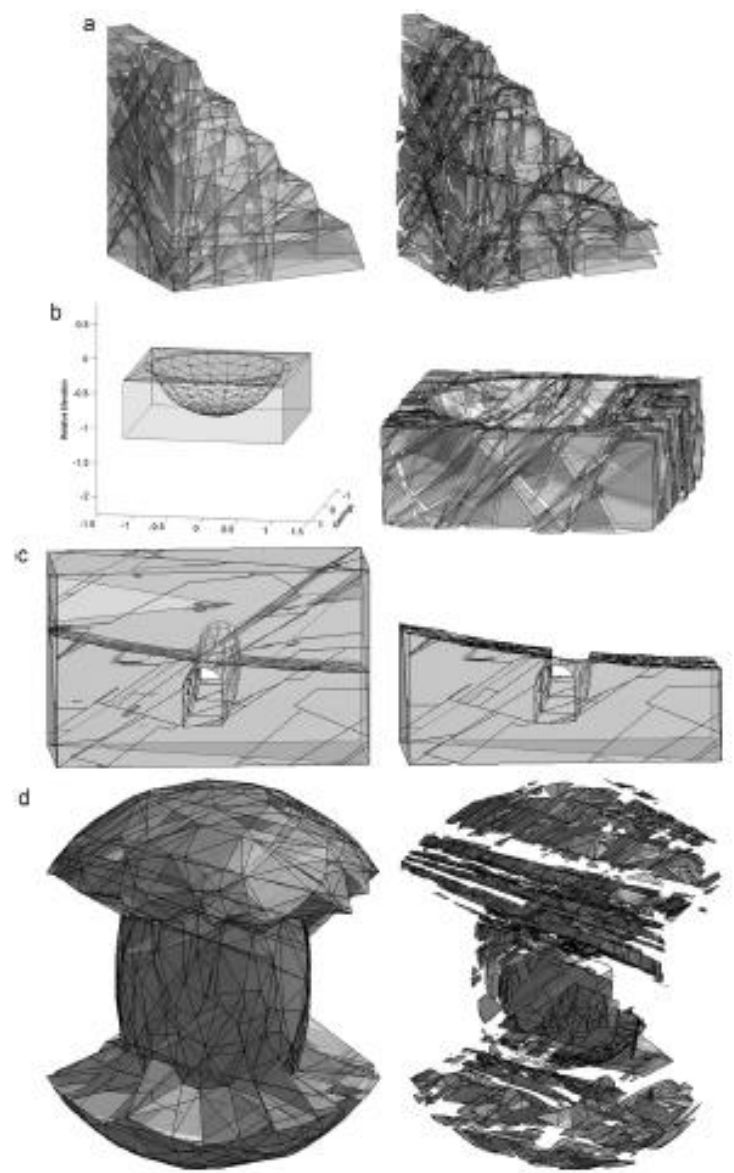

Figure 3 Example of polyhedral models (taken from Elmouttie et al., 2010). Shown are (a) open pit benches;

(b) bowl shaped excavation; (c) underground excavation; and (d) pillar

This form of analysis is sophisticated yet rapid enough to allow interrogation of the trustworthiness of the assumptions on which a model is based. For example, Figure 4 shows two differing predictions of potentially hazardous blocks in a coal mine high wall. Both predictions were based on identical structural mappings however one prediction assumes persistence of structures in the direction of bedding is twice that of the other. The difference in results is significant. Polyhedral modelling provides flexibility not available via simple wedge analysis to support interrogation of the effect of such assumptions on the mechanisms of block formation. 


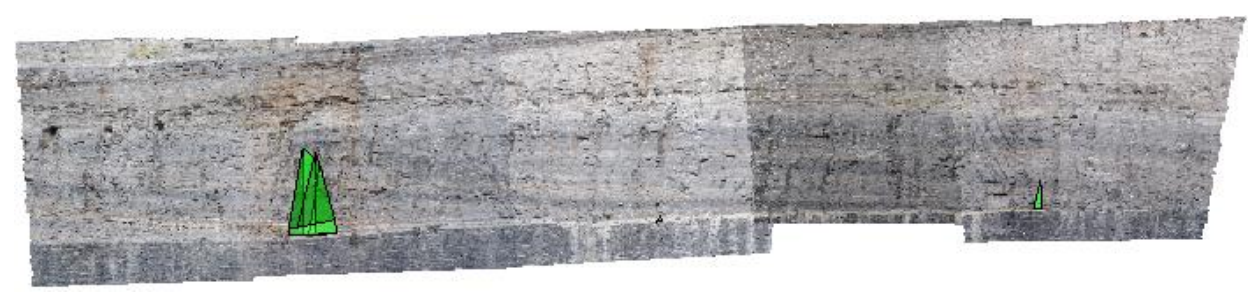

(a)

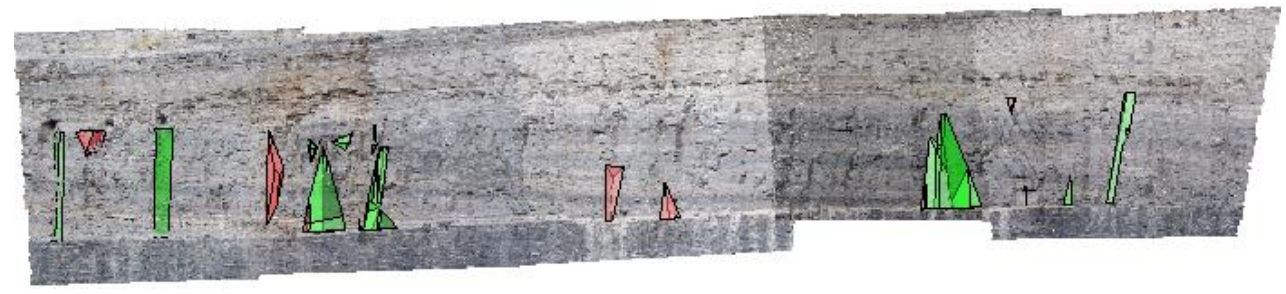

(b)

Figure 4 Differing predictions from rigid block stability analyses. Analysis shown in (a) assumes persistence of structures into the wall is half of that assumed in the analysis shown in (b)

\subsection{Quasi-stochastic analysis of uncertainty in digitally mapped structures}

Structures generated using the DFN method are typically defined in terms of being either deterministic (i.e. mapped) and stochastic (i.e. inferred). However, digital mapping methods provide the practitioner with the ability to quantify both the measurements as well as the model uncertainty associated with the mapped structures. This can be considered to be a quasi-stochastic analysis. The uncertainties associated with the mapped discontinuity properties can be significant, including orientation uncertainty (one to several degrees depending on the quality of the 3D data, structure curvature and the amount of exposure mapped) and size uncertainty (can be significant, particularly in sedimentary structures where persistence along bedding may differ significantly to that measured across bedding).

In Figure 5, the results of both a deterministic analysis and quasi-stochastic analyses for several scenarios considering differing amounts of orientation uncertainty are presented. These 'hazard maps' represent the likely location of daylighting blocks as predicted in the analysis shown in Figure 4 . The data corresponds to structural mappings on a coal mine highwall with bedding represented using stochastically generated weakness planes (see Elmouttie et al., 2013 for details). Uncertainty in the structure orientations has been gradually increased to determine the effect on the centre of mass of the daylighting blocks (hazards) and for each scenario, one hundred DFN realisations have been used to study the influence of this uncertainty. The colour scale used in the maps represents the percentage of realisations predicting daylighting blocks at particular locations. Even after $1^{\circ}$ standard deviation (normally distributed) as shown in the (b) map, the hazard maps begin to look markedly different, a reflection of the changing three-dimensional character of the block shape. The map shown in (d) corresponds to the scenario where the uncertainty in individual structure orientations is that of the structure sets themselves. The final map shown in (e) is presented for reference as it represents the most pessimistic scenario where almost no knowledge of the structure orientations is available. This type of analysis can therefore be used quantify the uncertainty associated with hazard characterisation based on the level of confidence one has in the input parameters. 


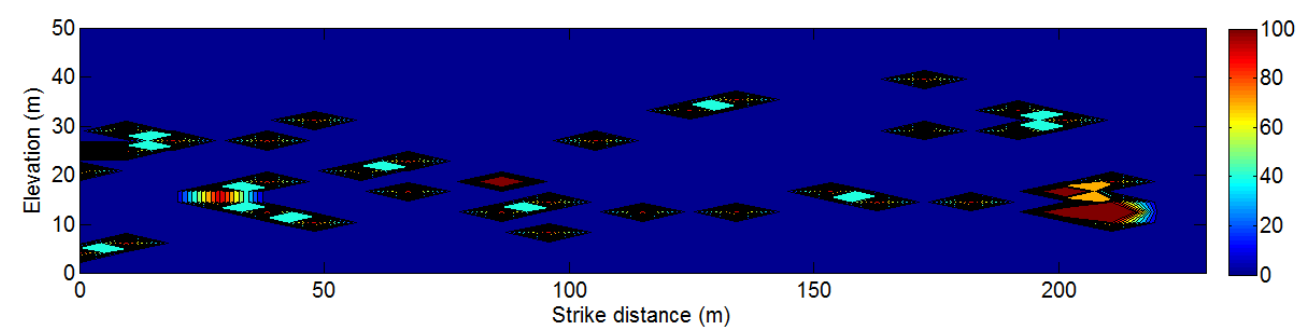

(a)

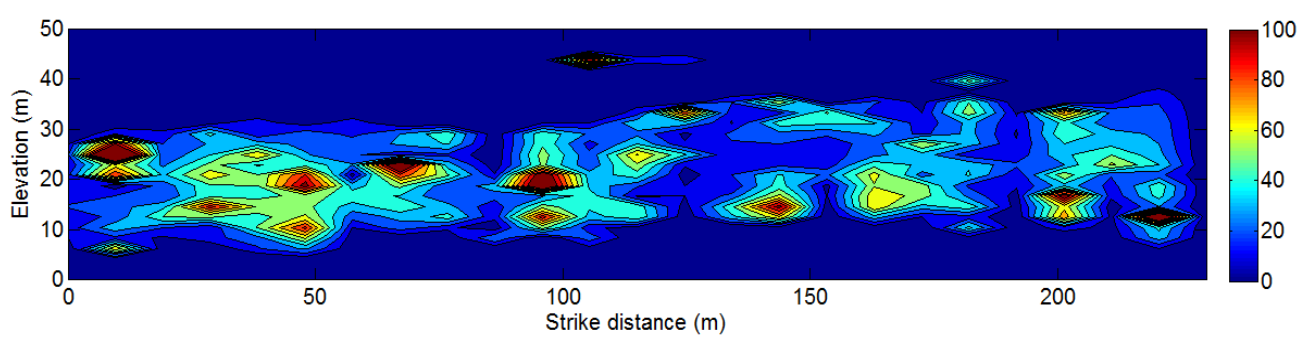

(b)

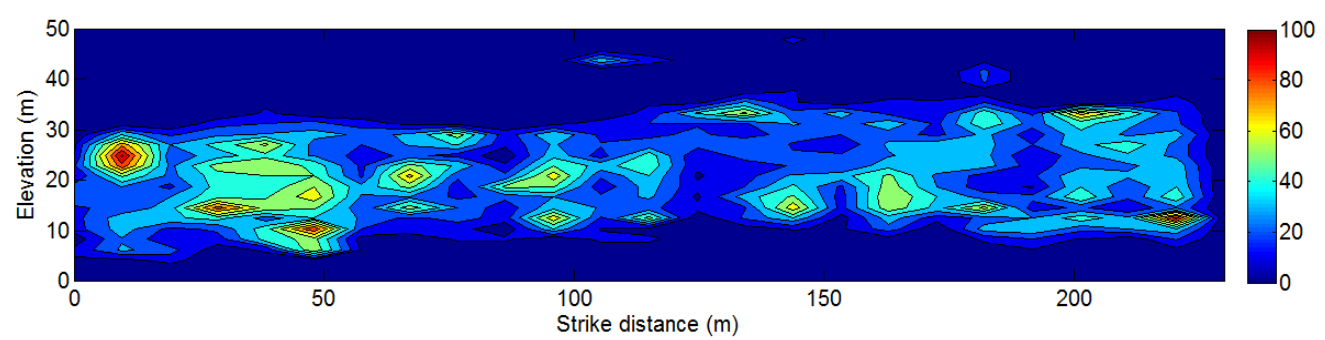

(c)

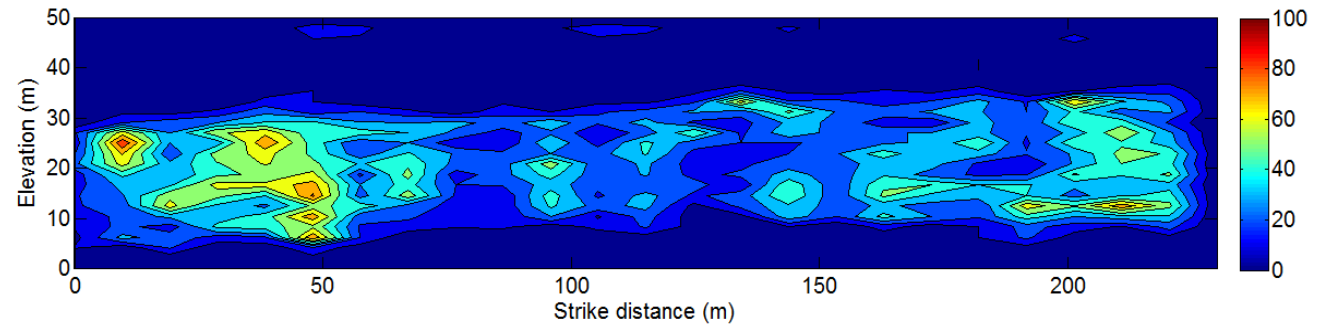

(d)

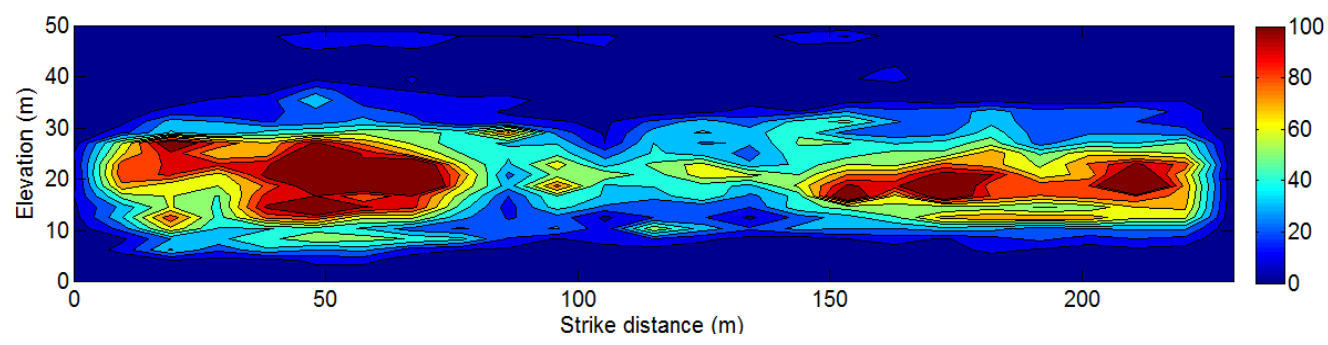

(e)

Figure 5 Hazard maps for orientation uncertainties in dip and dip direction of (a) $\left[0^{\circ} 0^{\circ}\right]$ (deterministic analysis);

(b) $\left[1^{\circ} 1^{\circ}\right]$ (most optimistic scenario); (c) $\left[2.5^{\circ} 5^{\circ}\right] ;$ (d) $\left[5^{\circ} 10^{\circ}\right]$ (set variance scenario); and (e) $\left[40^{\circ} 80^{\circ}\right]$

(most pessimistic scenario). Taken from Elmouttie et al. (2013) 
Of course, traditional DFN analysis can also be undertaken for more extensive characterisation of the uncertainty in the rock mass. However, depending on the scope of the work, such investigations may not necessarily satisfy the criterion of 'rapid characterisation'. The example shown in Figure 6 shows the analysis of predicted crest damage on a section of an open pit mine. Four structure sets were included in the DFN generation, with each realisation containing around 100,000 fractures. One hundred realisations were generated and the location and frequency of daylighting blocks was compiled in a hazard map. The colour scale used in Figure 6(b) represents the percentage of realisations predicting crest damage in the shear zone and therefore the reliability associated with this analysis.

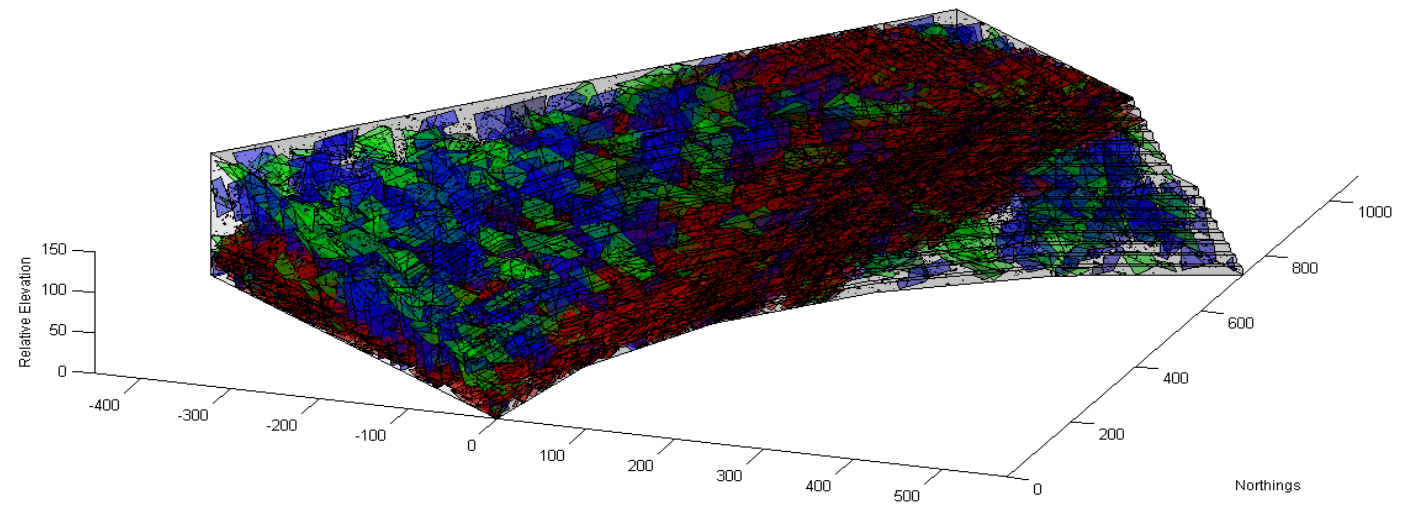

(a)

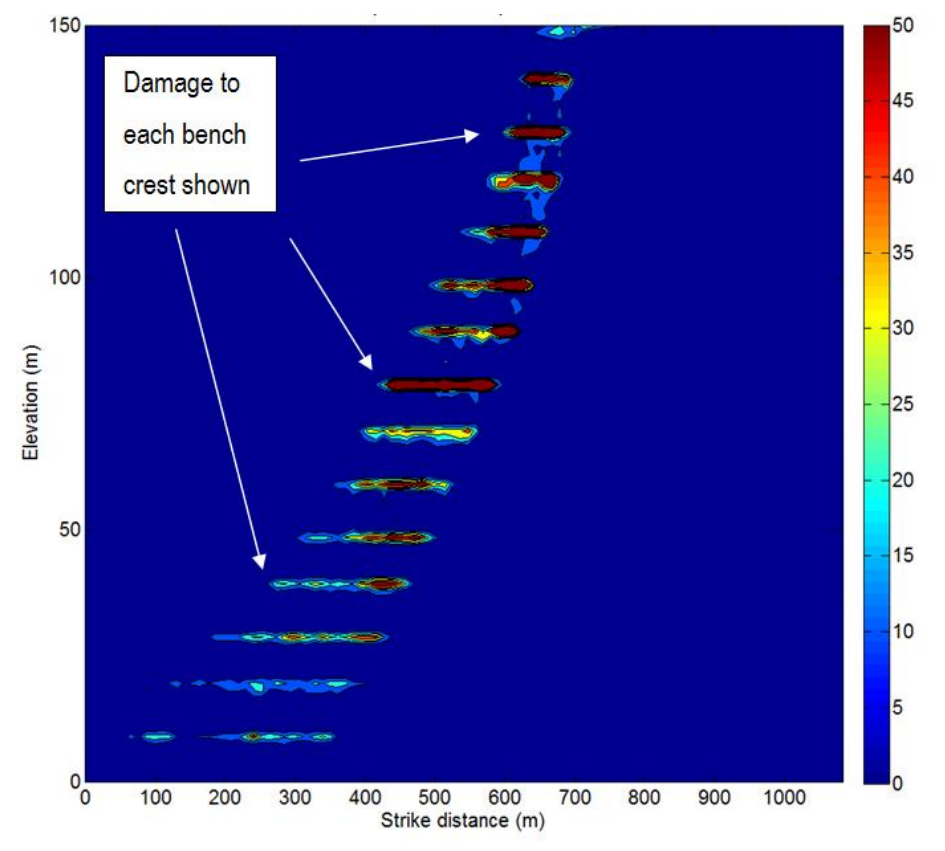

(b)

Figure 6 A DFN (a) and statistically based hazard map (b) for 15 benches of an open cut mine

\subsection{Summary}

A comparison of the relative merits of the techniques discussed is shown in Table 1. Only digital mapping combined with polyhedral modelling is sophisticated enough to adequately represent hazard frequency and location. 
Table 1 A comparison of stability analysis techniques

\begin{tabular}{|l|c|c|c|c|}
\hline & $\begin{array}{c}\text { Stereonet } \\
\text { Analysis }\end{array}$ & $\begin{array}{c}\text { Simple } \\
\text { Wedge } \\
\text { Analysis }\end{array}$ & $\begin{array}{c}\text { Digital Mapping } \\
\text { with Simple } \\
\text { Wedge } \\
\text { Detection }\end{array}$ & $\begin{array}{c}\text { Digital } \\
\text { Mapping with } \\
\text { Polyhedral } \\
\text { Modelling }\end{array}$ \\
\hline $\begin{array}{l}\text { Accounts for structure } \\
\text { orientation }\end{array}$ & $\checkmark$ & $\checkmark$ & $\checkmark$ & $\checkmark$ \\
\hline Accounts for structure size & $x$ & $\checkmark$ & $\checkmark$ & $\checkmark$ \\
\hline $\begin{array}{l}\text { Uses georeferenced data } \\
\text { Captures spatial distribution } \\
\text { of hazards across different } \\
\text { parts of the highwall }\end{array}$ & $x$ & $x$ & $\checkmark$ & $\checkmark$ \\
\hline $\begin{array}{l}\text { Adequately captures } \\
\text { frequency of hazards }\end{array}$ & $x$ & $x$ & $x$ & $\checkmark$ \\
\hline $\begin{array}{l}\text { Represents more realistic } \\
\text { block geometry (not just } \\
\text { simple wedges) }\end{array}$ & $x$ & $x$ & $x$ & $\checkmark$ \\
\hline $\begin{array}{l}\text { Unlimited numbers of } \\
\text { structure sets used to } \\
\text { predict block formation (not } \\
\text { limited to two) }\end{array}$ & $x$ & $x$ & $x$ & $\checkmark$ \\
\hline $\begin{array}{l}\text { Represents intersections of } \\
\text { minor structures (joints) } \\
\text { with major structures (e.g. } \\
\text { faults) }\end{array}$ & $x$ & $x$ & $x$ & $\checkmark$ \\
\hline $\begin{array}{l}\text { Non-planar structures (e.g. } \\
\text { curved faults) }\end{array}$ & $x$ & $x$ & & $\checkmark$ \\
\hline $\begin{array}{l}\text { Represents excavation, } \\
\text { geological and geotechnical } \\
\text { constraints }\end{array}$ & $x$ & $x$ & & $\checkmark$ \\
\hline
\end{tabular}

\section{$4 \quad$ Implementation}

In the following section, an approach that can be used to integrate the previously described methods with analysis of more complex failure mechanisms is discussed. A brief discussion of the use of multi-criteria evaluation (MCE) for more reliable hazard characterisation is then presented.

\subsection{Integration with more complex modelling}

Analysis of potential complex failure mechanisms requires more computationally intensive modelling techniques to capture rock mass failure. In the work reported in this paper, statistically based modelling of structurally controlled failures has been used to characterise the uncertainty associated with these hazards. Integration of these techniques can provide a means of quantifying uncertainty associated with more simplistic approaches as an approximation. A schematic representation of this approach is shown in Figure 7. Although modelling complex failure modes for a multitude of DFN realisations may not be 
computationally possible, using the polyhedral rigid block stability analysis to quantify the uncertainty associated with the rock mass characterisation and then analysing selected DFN realisation may be tractable (Elmouttie and Poropat, 2011).

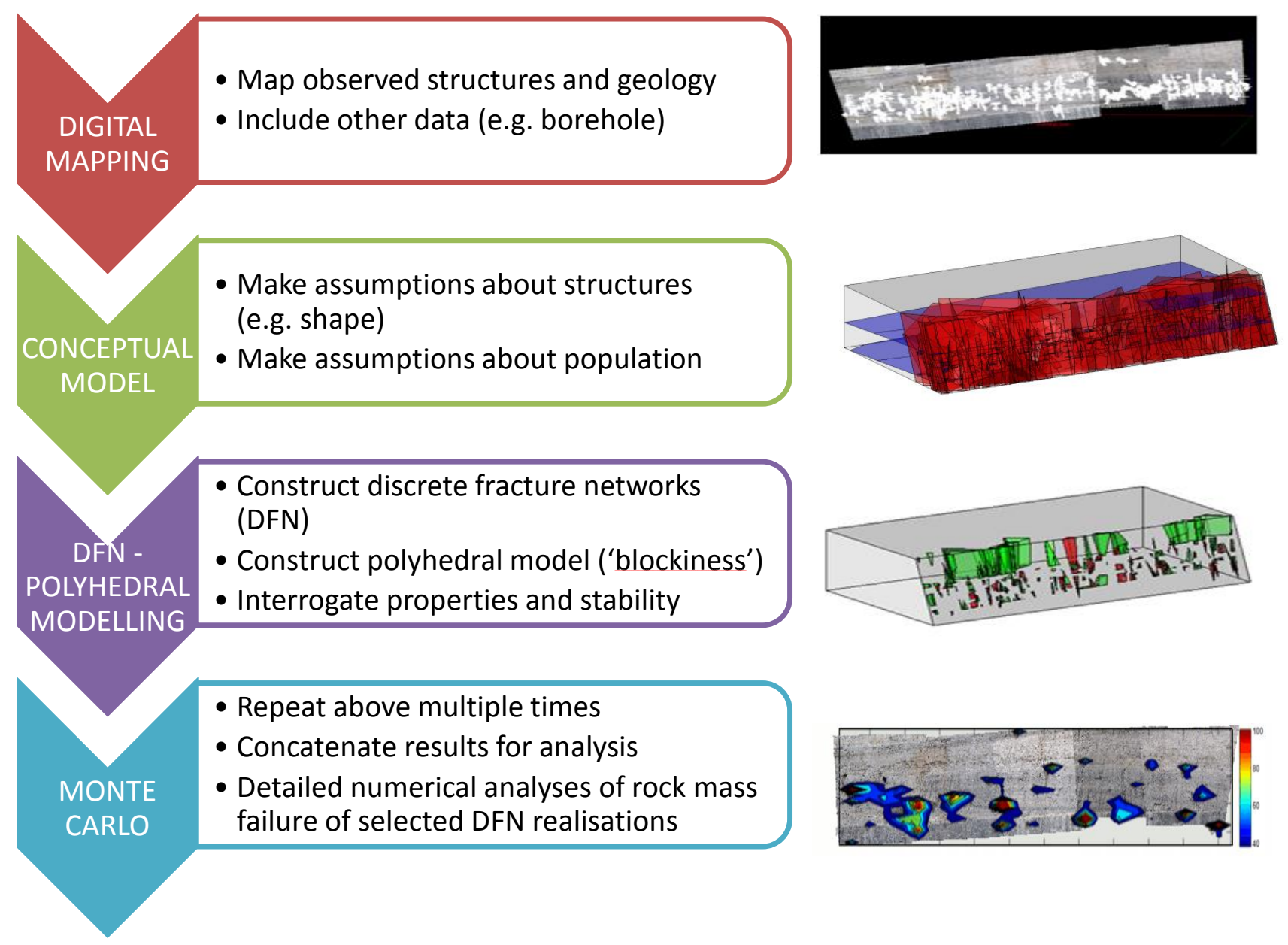

Figure 7 A schematic representation of the approach - integrating polyhedral based stability analysis with detailed analysis of complex failure mechanisms

\subsection{Multi-criteria evaluation}

The discussion presented in this paper has focussed on the use of structural data acquired via digital mapping of exposures. A variety of other data pertaining to characterisation of geotechnical hazards may be available (e.g. borehole data and geophysics, expert knowledge, inspection checklists etc.) and integrating these data is clearly desirable to mitigate model limitations, biases in data and 'blind-spots'. Combination of this data can be accomplished by using a MCE approach. Formal MCE methods originated in the 1960s and MCE has been integrated with geographic information systems (GIS) to assist decision making with the means to evaluate various alternatives on the basis of multiple and conflicting criteria or objectives (Carver, 1991).

MCE approaches are available in several software packages used in mining and civil engineering. Choosing appropriate weightings for the individual data 'layers' is a problematic part of this approach but this can be accomplished in different ways and formal methods for choosing methods have been defined in the literature (e.g. Malczewski, 1991). Figure 8 shows a schematic representation of this approach for hazard characterisation. Currently, the authors are investigating the use of MCE and similar methods for rapid characterisation of geotechnical hazards. 


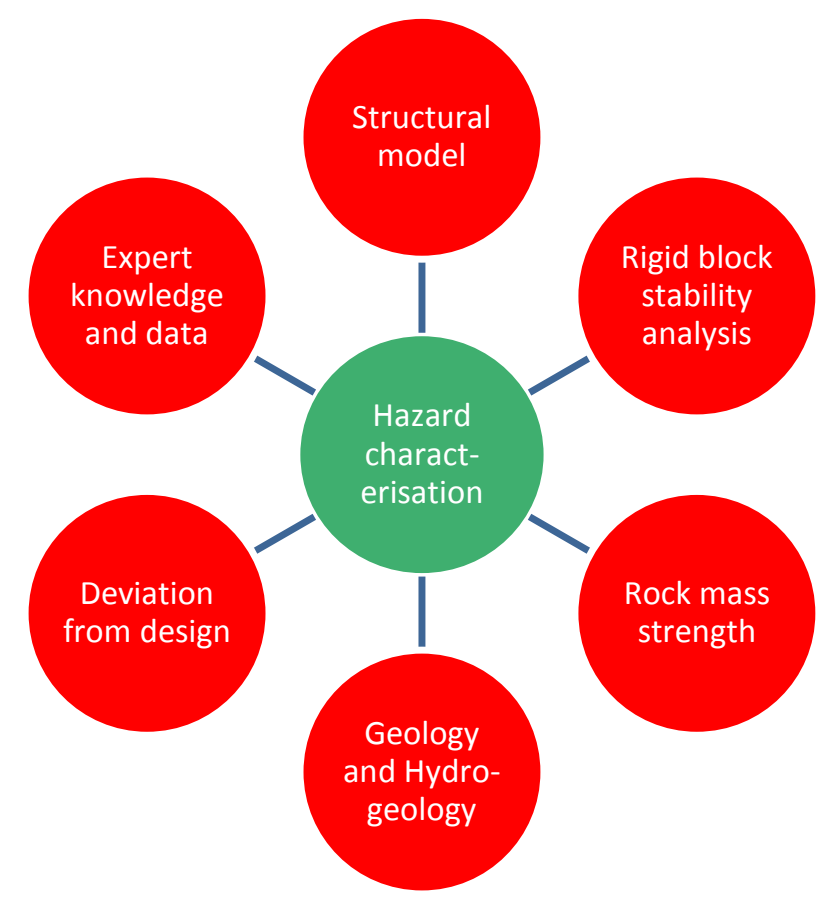

Figure 8 Integration of data from numerous sources to rapidly characterise hazards

\subsection{Field based implementation}

Field data and observations should be referenced continually during any computational analysis of potential failure mechanisms to ensure that the results of simulations align with reality as it may have been or is currently observed. Performing analysis whilst in the field is not always a practical option, however the algorithms described in this paper are suitable for implementation on field based computers (e.g. tablets).

With appropriate interface design, the practitioner can quickly interrogate scenarios and perform sensitivity analyses for identified hazards whilst observing those structures. This presence in the field can provide the practitioner with better understanding based on visual interpretation than can be achieved through digital photographs and is expected to stimulate a better appreciation of simplifications and uncertainties associated with the modelling process.

\section{$5 \quad$ Conclusions}

Rapid characterisation of hazards in open pit mining can be used alongside slope monitoring technologies to improve management and design decisions. Statistically based techniques can allow quantification of the uncertainty associated with predictions of structurally controlled failure mechanisms. These uncertainties can guide the practitioner as to the trustworthiness or reliability of analysis of more complex failure mechanisms.

\section{Acknowledgement}

The authors would like to acknowledge the support of the Australian Coal Association Research Program in the development of some of the algorithms used in elements of the structural modelling. The sponsors of the Large Open Pit Mine Slope Stability Project, which is managed by the CSIRO, are acknowledged for their support in developing applications to utilise algorithms for the analysis of open pit slope stability. The efforts of Shivakumar Karekal, Manoj Khanal and the two anonymous reviewers are also appreciated. 


\section{References}

Baecher, G.B., Lanney, N.A. and Einstein, H.H. (1978) Statistical description of rock properties and sampling, in Proceedings 18 th U.S. Symposium on Rock Mechanics, 22-24 June 1977, Keystone, USA, Johnson Publisher, Golden, USA.

Billaux, D., Chiles, J.P., Hestir, K. and Long, J.C.S. (1989) Three dimensional statistical modelling of a fractured rock mass - an example from the Fanay-Augeres Mine, International Journal of Rock Mechanics and Mining Sciences \& Geomechanics Abstracts, Elsevier, Vol. 26(3/4), pp. 281-299.

Carver, S. (1991) Integrating multi-criteria evaluation with geographic information systems, International Journal of Geographical Information Systems, Taylor \& Francis, Vol. 5, pp. 321-339.

Dershowitz, W.S. (1984) Rock joint systems, PhD Thesis, Massachusetts Institute of Technology, Boston, USA.

Elmouttie, M., Poropat, G.P. and Guest, A. (2008) On the need for polyhedral representation of blocky rock masses, in Proceedings First Southern Hemisphere International Rock Mechanics Symposium (SHIRMS), Y. Potvin, J. Carter, A. Dyskin and R. Jeffrey (eds), Vol. 2 - Fundamental and Petroleum, 16-19 September 2008, Perth, Australia, Australian Centre for Geomechanics, Perth, pp. 433-446.

Elmouttie, M., Poropat, G. and Hamman, E.C.F. (2009) Simulations of the sensitivity of rock structure models to field mapped parameters, in Proceedings International Symposium on Rock Slope Stability in Open Pit Mining and Civil Engineering (Slope Stability 2009), 9-11 November 2009, Santiago, Chile, CD-rom only.

Elmouttie, M., Poropat, G. and Krahenbuhl, G. (2010) Polyhedral modeling of rock mass structure International Journal of Rock Mechanics and Mining Sciences \& Geomechanics, Elsevier, Vol. 47(4), pp. 544-552.

Elmouttie, M.K. and Poropat, G.V. (2011) Uncertainty propagation in structural modelling, in Proceedings International Symposium on Rock Slope Stability in Open Pit Mining and Civil Engineering (Slope Stability 2011), 18-21 September 2011, Vancouver, Canada, Canadian Rock Mechanics Association, Canada, CD-rom only.

Elmouttie, M.K., Krähenbuhl, G., Poropat, G.V. and Kelso, I. (2013) Stochastic representation of sedimentary geology, Rock Mechanics and Rock Engineering, DOI: 10.1007/s00603-013-0411-x, Springer Verlag.

Jing, L. and Stephansson, O. (1994) Topological identification of block assemblages for jointed rock masses, International Journal of Rock Mechanics and Mining Sciences \& Geomechanics Abstracts, Elsevier, Vol. 31, pp. 163-172.

Jing, L. (2000) Block system construction for three dimensional discrete element models of fractured rocks, International Journal of Rock Mechanics and Mining Sciences \& Geomechanics, Elsevier, Vol. 37, pp. 645-659.

Jing, L. (2003) A review of techniques, advances and outstanding issues in numerical modelling for rock mechanics and rock engineering, International Journal of Rock Mechanics \& Mining Sciences, Elsevier, Vol. 40, pp. 283-353.

Lin, D., Fairhurst, C. and Starfield, A.M. (1987) Geometrical identification of three dimensional rock block systems using topological techniques, International Journal of Rock Mechanics and Mining Sciences \& Geomechanics Abstracts, Elsevier, Vol. 24(6), pp. 331-338.

Lu, J. (2002) Systematic identification of polyhedral rock blocks with arbitrary joints and faults, Computers and Geotechnics, Elsevier, Vol. 29, pp. 49-72.

Menéndez-Díaz, A., González-Palacio, C., Álvarez-Vigil, A.E., González-Nicieza, C. and Ramírez-Oyanguren, P. (2009) Analysis of tetrahedral and pentahedral key blocks in underground excavations, Computers and Geotechnics, Elsevier, Vol. 36, pp. 1009-1023.

Malczewski, J. (1991) GIS and Multicriteria Decision Analysis, John Wiley \& Sons, 393 p.

Rogers, S.F., Kennard, D.K., Dershowitz, W.S. and Van As, A. (2007) Characterising the in situ fragmentation of a fractured rock mass using a discrete fracture network approach, in Proceedings Rock Mechanics: Meeting Society's Challenges and demands, E. Eberhardt, D. Stead, T. Morrison (eds), 1st Canada-US Rock Mechanics Symposium, 27-31 May 2007, Vancouver, Canada, Taylor \& Francis Group, London.

Stead, D., Eberhardt, E. and Coggan, J.S. (2006) Developments in the characterisation of complex rock slope deformation and failure using numerical modelling techniques, Engineering Geology, Elsevier, Vol. 83, pp. 217-235.

Wang, L.G., Yamashita, S., Sugimoto, F., Pan, C. and Tan, G. (2003) A Methodology for Predicting the In Situ Size and Shape Distribution of Rock Blocks, Rock Mechanics and Rock Engineering, Springer, Vol. 36(2), pp. 121-142. 